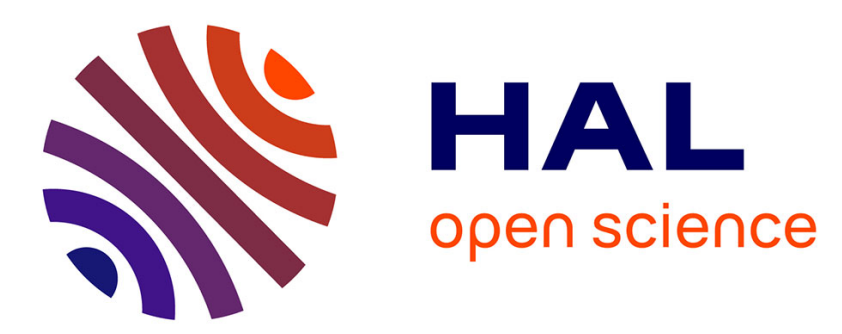

\title{
Foaming of binary mixtures: link with the nonlinear behaviour of surface tension in asymmetric mixtures
} H P Tran, L Delance, N Passade-Boupat, E Verneuil, F Lequeux, L Talini

\section{To cite this version:}

H P Tran, L Delance, N Passade-Boupat, E Verneuil, F Lequeux, et al.. Foaming of binary mixtures: link with the nonlinear behaviour of surface tension in asymmetric mixtures. Langmuir, 2021, 10.1021/acs.langmuir.1c02198 . hal-03419871

\section{HAL Id: hal-03419871 \\ https://hal-espci.archives-ouvertes.fr/hal-03419871}

Submitted on 8 Nov 2021

HAL is a multi-disciplinary open access archive for the deposit and dissemination of scientific research documents, whether they are published or not. The documents may come from teaching and research institutions in France or abroad, or from public or private research centers.
L'archive ouverte pluridisciplinaire HAL, est destinée au dépôt et à la diffusion de documents scientifiques de niveau recherche, publiés ou non, émanant des établissements d'enseignement et de recherche français ou étrangers, des laboratoires publics ou privés. 


\title{
Foaming of binary mixtures: link with the nonlinear behaviour of surface tension in asymmetric mixtures
}

\author{
H.P. $\operatorname{Tran}^{1,2}$, L. Delance ${ }^{1,2}$, N. Passade-Boupat ${ }^{2,3}$, E. Verneuil ${ }^{1,2}$, F. Lequeux ${ }^{1,2}$ and L. Talini ${ }^{4 *}$ \\ ${ }^{1}$ CNRS, Sciences et Ingénierie de la Matière Molle, ESPCI Paris, PSL Research University, Sorbonne Université, \\ 75005 Paris, France. \\ 2 Laboratoire Physico-Chimie des Interfaces Complexes, ESPCl, 10 rue Vauquelin, 75005 Paris, Bâtiment \\ CHEMSTARTUP, Route Départementale 817, 64170 Lacq, France. \\ 3 Total S.A. 64170 Lacq, France. \\ ${ }^{4}$ CNRS, Surface du Verre et Interfaces, Saint-Gobain, 93300 Aubervilliers,France \\ *e-mail: laurence.talini@espci.fr
}

\begin{abstract}
The lifetimes of single bubbles or foams that are formed in mixtures of liquids can be several orders of magnitude larger than the ones formed in pure liquids. We recently demonstrated that this enhanced stability results from differences between bulk and interfacial concentrations in the mixture, which induce a thickness dependence of the surface tension in liquid films, and thus a stabilizing Marangoni effect. Concentration differences may be associated with nonlinear variations of surface tension with composition and we further investigate their link with foamability of binary mixtures. We show that, for asymmetric binary mixtures, i.e. made of molecules of very different sizes, strong nonlinearities in surface tension can be measured, that are associated with large foam lifetimes. When the molecules that occupy the largest surface areas have the smallest surface tension, the surface tension of the mixture varies sublinearly with composition, reflecting an enrichment in this species at the interface with air, as classically reported in the literature. In contrast, when they exhibit the largest surface tension, superlinear variations of surface tension are observed despite a similar enrichment. We discuss these variations in light of a simple thermodynamic model for ideal mixtures and we demonstrate why foam stability is enhanced for both sublinear and superlinear surface tension variations, thus shedding new light on foamability without added surfactants.
\end{abstract}

\section{INTRODUCTION}

More than a century ago, it was observed that the surface tensions of some binary mixtures do not vary linearly with their composition ${ }^{1}$. For instance, it is well known that the surface tension of water/alcohol mixtures is smaller than the one predicted by a linear variation with composition. This effect was further ascribed to the presence of a surface layer of composition different from the one of the bulk; in the case of water/alcohol mixtures, the surface layer is enriched in alcohol, which has a smaller surface tension than water, resulting in sublinear variations with composition of the surface tension of the mixture. Thermodynamical models were developed in that framework ${ }^{2}$ and the detailed description of this effect remains a current research subject ${ }^{3}$, as well as the properties of the surface layer ${ }^{4}$.

In parallel, it has been shown that some binary mixtures have foaming properties: whereas the lifetimes of bubbles formed in pure low-viscosity liquids is expected to be smaller than $1 \mathrm{~ms}$ in the absence of contaminants ${ }^{5}$, the lifetimes of bubbles in binary mixtures of similar viscosities can reach several tens of seconds ${ }^{6}$ and transient foams are observed. Consequences of the foaming behavior of liquid mixtures impact multiple applications in which control of foaming is needed; when foaming is 
desired it provides a way to produce foams without adding surfactants by simply mixing different liquids. In contrast, when unwanted, foaming of mixtures can be detrimental to applications; for instance, it impedes lubrication by oils in car gear boxes.

We have recently demonstrated that the enhanced stability of foams in binary mixtures results from the differences in concentrations at the interface and in the bulk ${ }^{7}$, that are associated with nonlinear surface tensions. Because of these differences, the increase of surface to volume ratio of liquid films that occurs during the stage of foam formation requires changes in both surface and bulk concentration. As a result, the surface tension of a liquid film increases with decreasing thicknesses. This effect induces a slowing down of the drainage of the liquid films between bubbles in foams, and thus increases the lifetimes of these films.

Our previous experiments were conducted with mixtures of molecules with similar sizes ${ }^{7}$. In that case, because the surfaces of molecules have close values, the variations of surface tension with composition simply reflect the population at the interface with air. In contrast, for mixtures of molecules with very different surfaces, hereafter called asymmetric, the situation is more complex. Here, we investigate the link between foaming and surface tension variations of mixtures of molecules with a large size ratio, which can present some counter-intuitive behaviour. We discuss them in light of the ideal solution approximation for surface tension of binary mixtures.

\section{EXPERIMENTAL SECTION}

Materials. The binary mixtures were made with $n$-heptane, $n$-decane, $n$-hexadecane and toluene (all with purity $>99 \%$, Sigma-Aldrich), as well as Polydimethylsiloxane (PDMS, ABCR, viscosity 5cSt). Experiments were also conducted with $n$-eicosane ( $>99 \%$, Sigma-Aldrich), which has a melting point at $36.65^{\circ} \mathrm{C}$. The measurements made with eicosane were performed at $50^{\circ} \mathrm{C}$ whereas all other measurements were made at room temperature. Liquids were mixed and stirred to obtain homogeneous mixtures with different compositions. We list in Table 1 the properties of the used binary mixtures $A / B, A$ being the species with the smallest surface tension. In particular, we report the volume ratio of the mixture which we define as the ratio of the molar volume of species $B, v_{B}$ and the molar volume of $A, v_{A}$. The surface areas $\sigma_{A}$ and $\sigma_{B}$ occupied in the gas/liquid interface by respectively molecules $A$ and $B$ are expressed as molar surfaces; in a first approximation, they are computed from molar volumes as the areas of cuboid molecules.

\begin{tabular}{|c|c|c|c|c|c|c|c|c|c|c|c|}
\hline \multirow[t]{2}{*}{ Mixture $A / B$} & \multicolumn{2}{|c|}{$\begin{array}{l}\text { Surface } \\
\text { tensions } \\
\left(\mathrm{mN} \cdot \mathrm{m}^{-1}\right)\end{array}$} & \multicolumn{2}{|c|}{$\begin{array}{c}\text { Molar } \\
\text { volumes } \\
\left(10^{-4}\right. \\
\left.\mathrm{m}^{3} \cdot \mathrm{mol}^{-1}\right)\end{array}$} & \multirow{2}{*}{$\begin{array}{c}\begin{array}{c}\text { Volu } \\
\text { me } \\
\text { ratio }\end{array} \\
v_{\mathrm{B}} / v_{\mathrm{A}}\end{array}$} & \multicolumn{2}{|c|}{$\begin{array}{c}\text { Cuboid } \\
\text { molar } \\
\text { surfaces } \\
\left(10^{6}\right. \\
\left.\mathrm{m}^{2} \cdot \mathrm{mol}^{-1}\right) \\
\end{array}$} & \multirow{2}{*}{$\begin{array}{c}\begin{array}{c}\text { Cuboid } \\
\text { surface } \\
\text { ratio }\end{array} \\
\sigma_{\mathrm{B}} / \sigma_{\mathrm{A}}\end{array}$} & \multirow{2}{*}{$\begin{array}{c}\begin{array}{c}\text { Best } \\
\text { surface } \\
\text { ratio }\end{array} \\
\sigma_{\mathrm{B}} / \sigma_{\mathrm{A}}\end{array}$} & \multirow{2}{*}{$\begin{array}{c}\frac{\sigma_{A}\left(\gamma_{B}-\gamma_{A}\right)}{R T} \\
\mathrm{~S}_{\mathrm{A}}\end{array}$} & \multirow{2}{*}{$\begin{array}{c}\frac{\sigma_{B}\left(\gamma_{B}-\gamma_{A}\right)}{R T} \\
\mathrm{~S}_{\mathrm{B}}\end{array}$} \\
\hline & $\gamma_{\mathrm{A}}$ & $\gamma_{\mathrm{B}}$ & $v_{\mathrm{A}}$ & $v_{\mathrm{B}}$ & & $\sigma_{\mathrm{A}}$ & $\sigma_{\mathrm{B}}$ & & & & \\
\hline PDMS/Decane & 18.8 & 23.3 & 8.39 & 1.96 & 0.23 & 0.75 & 0.29 & 0.38 & 0.2 & 1.36 & 0.53 \\
\hline Octane/Toluene & 21.4 & 27.9 & 1.64 & 1.07 & 0.65 & 0.25 & 0.19 & 0.75 & 0.4 & 0.66 & 0.50 \\
\hline $\begin{array}{l}\text { Decane/Eicosane } \\
\left(50^{\circ} \mathrm{C}\right)\end{array}$ & 21.0 & 26.5 & 2.01 & 3.67 & 1.86 & 0.29 & 0.43 & 1.49 & 2 & 0.64 & 0.96 \\
\hline Heptane/Hexadecane & 19.8 & 27.2 & 1.47 & 2.94 & 1.99 & 0.24 & 0.37 & 1.58 & 3 & 0.72 & 1.11 \\
\hline
\end{tabular}

Table 1: Used liquids and their properties. The surface tension values were measured and the molar volumes found in the literature. The molar surfaces were computed from the molar volumes in the 
cuboid molecule approximation. The best surface ratio are the values for which the best agreement was found between the experimental and predicted variations of surface tension with the ones predicted by Butler's equation (eq. (13)).

Tensiometry. The surface tensions of all binary mixtures were measured as a function of their composition with a tensiometer (Teclis) that measured the shapes of rising bubbles formed in mixtures. We have found that, in all mixtures, a constant value of surface tension is reached instantaneously, indicating relaxation times smaller than the time resolution of the tensiometer $(0.1$ s). Measurement conducted over long times (up to 1 hour) did not show any variation of surface tension showing evaporation is negligible. The densities of the mixtures were computed in the approximation of volume additivity that has been validated in previous studies for some mixtures ${ }^{8}$. This assumption was checked for the other mixtures by weighing a given volume of liquid.

Viscosimetry. The viscosities of PDMS/decane and octane/toluene mixtures were measured (Rheometer Low Shear 400, Lamy rheology). The viscosities of alkane mixtures were computed using the empirical Kendall-Monroe equation ${ }^{9}$.

Foaming experiments. Bikerman columns constituted by a glass column of diameter $2 \mathrm{~cm}$ with a porous glass filter at the bottom (Robu, porosity: 10-16 $\mu \mathrm{m}$ ), were used to form foams and measure their lifetimes ${ }^{10}$. The column was filled with the binary mixture up to a height of $10 \mathrm{~cm}$. Air pumped in a closed loop was injected at the bottom of the column at a flowrate corresponding to the average velocity $V_{\text {gas }}=1.9 \times 10^{-2} \mathrm{~m} . \mathrm{s}^{-1}$. The PDMS/decane mixture strongly foams and a much smaller gas velocity was used, $V_{\text {gas }}=2 \times 10^{-3} \mathrm{~m} . \mathrm{s}^{-1}$. Evaporation effects are expected to destabilize foams in all mixtures except hexane-hexadecane ${ }^{7}$, and larger foam heights were accordingly measured with negligible evaporation, i.e. in the closed loop in which air saturates in volatile species. In order to prevent retention of PDMS in the porous filter, it was chemically modified using a perfluorosilane. Briefly, the inner column was set to low pressure, and an air plasma was generated for $60 \mathrm{~s}$. A droplet of perfluorotrichlorosilane was then evaporated at low vapour pressure inside the column leading to chemical modification of the glass filter and no imbibition of the filter by the PDMS/decane mixture. The bubble radius in the formed foams was found to slightly depend on the liquid mixture but nevertheless remained within the range 500-800 $\mu \mathrm{m}$. During gas injection, a foam forms at the top of liquid, as shown in Fig. 1. Its height reaches a stationary value $H$ within a few seconds. The lifetime of the foam we define is the ratio of the foam height and the average gas injection velocity, $H / V_{\text {gas }}$. We have checked that the height varied linearly with injection velocity in the investigated flowrate range and did not depend on the initial liquid height in the column.

In order to compare the foaming properties of mixtures of different viscosity $\mu$ and surface tension $\gamma$ on which lifetimes depend, it is convenient to consider the product of average bubble lifetime and capillary velocity $\gamma / \mu$

$$
L_{\tau}=\frac{\gamma H}{\mu V_{\text {gas }}}
$$

For all mixtures, $L_{\tau}$ is independent of injection conditions within the investigated range. 


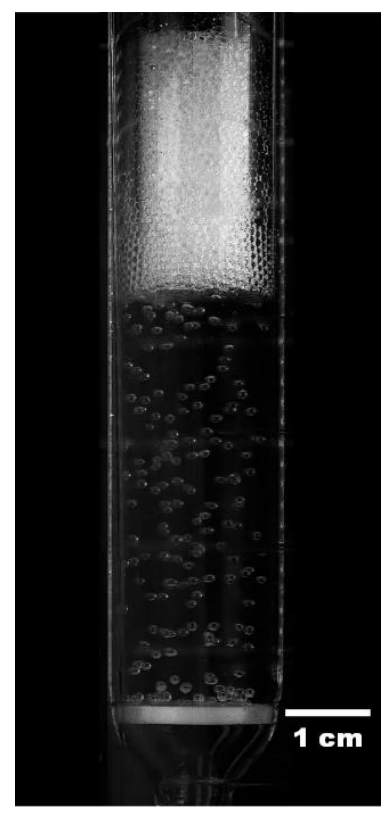

Fig. 1: Foam formed in a Bikerman column. The white part at the bottom of the column is the porous filter through which vapor-saturated air is injected.

\section{RESULTS}

Surface tension of mixtures. The reduced surface tensions of four A/B mixtures are shown in Fig. 2 as a function of the molar fraction of the species $A$ that has the smallest surface tension. Two different behaviors are observed. On the one hand, in both the PDMS/decane and octane/toluene mixtures, the surface tension exhibits sublinear variations with composition. It corresponds to a surface composition enriched in the species with the smallest surface tension, which has also the largest molar volume, i.e. either PDMS or octane. Similar variations have been widely reported with mixtures of different natures. ${ }^{3 b, 8,11}$ Clearly, the sublinearity is more marked for the PDMS/decane, which has the largest surface ratio. On the other hand, the surface tensions of heptane/hexadecane and decane/eicosane vary superlinearly with their compositions. This effect has been scarcely evidenced up to date, it is observed when the species with the smallest surface tension has the smallest molar volume, yielding surface ratios larger than unity, consistently with previous findings ${ }^{12}$. We will show in the following that the superlinearity of surface tension in these mixtures results from the same effect as in mixtures with sublinear variations of surface tension; in both cases, the concentrations at the interfaces differ from the ones in the bulk, the species with the smallest surface tension is always more concentrated at the surface than in the bulk. The sign of the nonlinearity depends on the surface ratio of the molecules. 


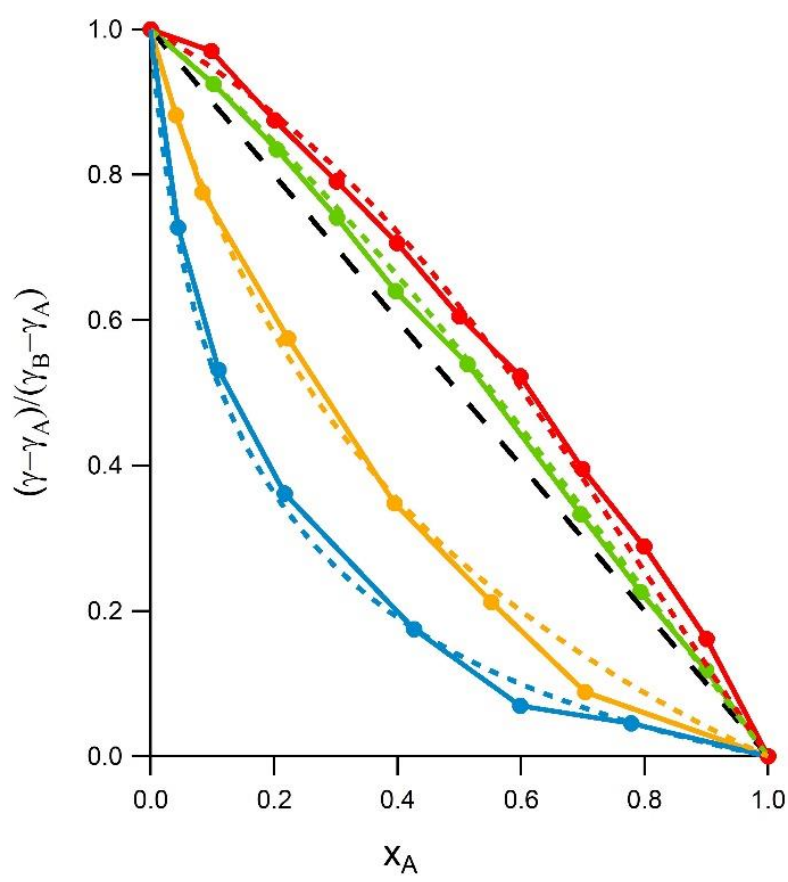

Fig. 2: Normalized surface tensions of binary mixtures $A / B$ as a function of the molar fraction of $A$, the species with the smallest surface tension. From left to right with full line and markers: PDMS/decane (blue), octane/toluene (orange), decane/eicosane (green) and heptane/hexadecane (red). The dashed line indicates linear variations. The dotted lines show the surface tensions computed from eq. (13) with the liquid parameters and the adjusted surface ratios given in Table 1. All measurements were made at room temperature except the ones with the decane/eicosane mixture that were performed at $50^{\circ} \mathrm{C}$.

Lifetimes of foams in mixtures. We have found that foam forms during Bikermann tests with all investigated mixtures. The measured lifetimes are multiplied by the capillary velocity in the same mixture, in order to account for viscosity and surface tension effects following eq. (1). The resulting values $L_{\tau}$ are shown in Fig. 3 as a function of volume fraction of the species with the smallest surface tension. The values of $L_{\tau}$ range from a few tens to a few hundreds of meters. They are therefore larger by more than $10^{4}$ than the bubble size in the formed foams. Very large typical lengths were similarly found to be involved in the bursting of bubbles at the surface of tap water ${ }^{5}$, and are currently not understood. However, $L_{\tau}$ provides a convenient way to compare lifetimes obtained in mixtures with different viscosities and surface tensions, and we will consider it as a normalized lifetime rather than a physical length.

All curves of Fig. 3 exhibit a maximum at a composition that depends on the asymmetry of the mixture. For smaller values of the volume ratios, corresponding to sublinear variations of surface tension, the position of the maximum is reached for $x_{A}<0.5$. In contrast, it corresponds to $x_{A}>0.5$ for surface ratios larger than unity, for which superlinear variations of surface tension are observed. However, there is no correlation between the values of the volume ratio and the amplitude of the maximum $L_{\tau}$. In particular, we have found the PDMS/decane mixture formed foams with very large lifetimes yielding very large values of $L_{\tau}$. The position of the maximum in the latter curve is also reached for a strikingly small $\left(\sim 10^{-3}\right)$ molar fraction in PDMS. Remarkably, PDMS is widely used as an antifoaming agent in aqueous solutions ${ }^{13}$ whereas it clearly promotes foaming when added to decane, with which it is miscible. We will show later that the profoaming property of PDMS added to decane results from both the rather large difference between their surface tensions and the large volume ratio of the two molecules. 


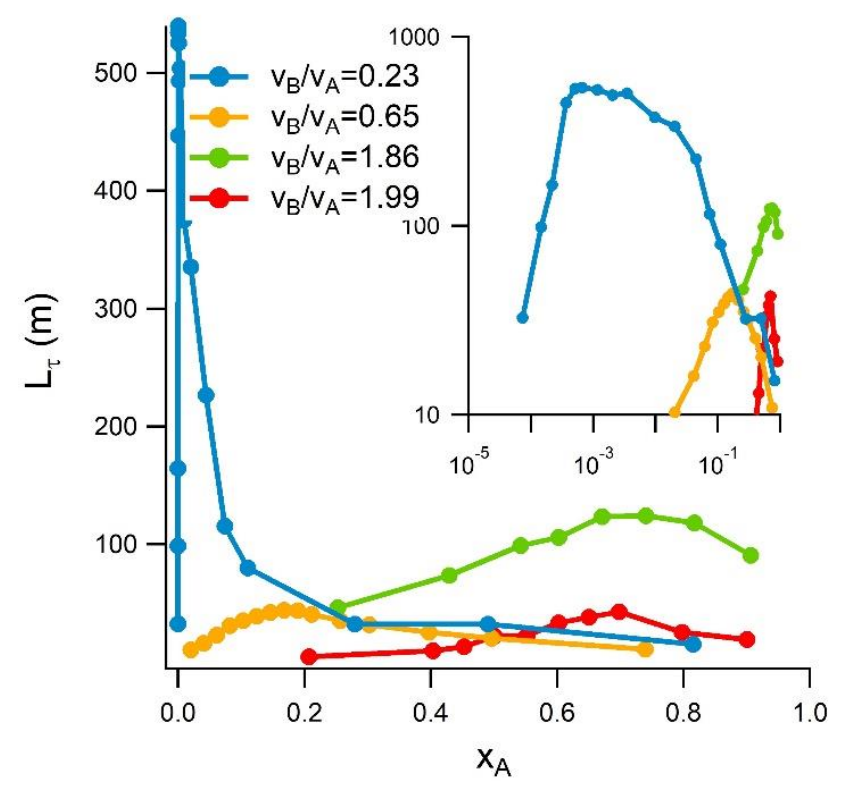

Fig. 3: Product $L_{\tau}$ of measured lifetimes and capillary velocities computed following eq. (1), obtained in the same mixtures as in Fig. 2 and as a function of the molar fraction of the species with the smallest surface tension. All experiments were performed at room temperature $\left(20^{\circ} \mathrm{C}\right)$ but the one with decane/eicosane conducted at $50^{\circ} \mathrm{C}$. Inset: same curves in log-log scale.

Thickness-dependent surface tension. We have shown that the differences in bulk and surface concentrations in binary mixtures result in a thickness dependence of the interfacial tension ${ }^{7}$. Actually, since thinning implies an increase of surface to volume ratio, a thin liquid film cannot further thin down without modifying its bulk and surface concentrations. Consequently, thinning implies a decrease of the surface concentration of species $A$, and therefore an increase of surface tension, which itself opposes thinning. As a liquid film forms in the foam between two bubbles coming closer, in a first and fast stage it is stretched without significant drainage, i.e. at constant volume. Its surface to volume ratio increases and, as a consequence of the partition of species between bulk and interfaces, the surface tension $\gamma_{f}$ in its flat part increases with decreasing thicknesses $h$ following

$$
\gamma_{f}(h)-\gamma=\gamma \frac{\alpha}{h}
$$

Where $\gamma$ is the surface tension of the liquid mixture as measured in a tensiometry experiment, and $\alpha$ a microscopic length that is derived in the following.

The resulting thickness-dependent interfacial tension allows a transient mechanical equilibrium of the film connected to a meniscus, called a Plateau border in foams. As detailed in a previous publication ${ }^{7}$ and schematized in Fig. 4, equilibrium of the tensions in the film is reached because the tension is larger in the flat part than in the curved part of the film. Although the increase in surface tension in the flat part is very small (less than 1\%), it allows the film tension, i.e. the sum of surface tension and pressure times thickness, to be balanced between the flat and curved parts of the film. We emphasize no equilibrium could be reached without the increase of surface tension. Once the equilibrium is reached for a given shape of the film, the pressure is nevertheless smaller in the Plateau border than in the film and the liquid is further sucked from the film to the Plateau border. The lifetimes of the liquid films are determined by the duration of film drainage, which is a relatively slow process (typically seconds) ${ }^{14}$. This duration depends itself on the initial film geometry (i.e. on the curvature radius of the Plateau border), and is observed to vary proportionally with the length $\alpha .^{7}$ This is why the relation 
between partition of molecules at the interface and in the bulk, and its role on the interfacial tension is crucial to understand the lifetime of foams formed in mixtures.

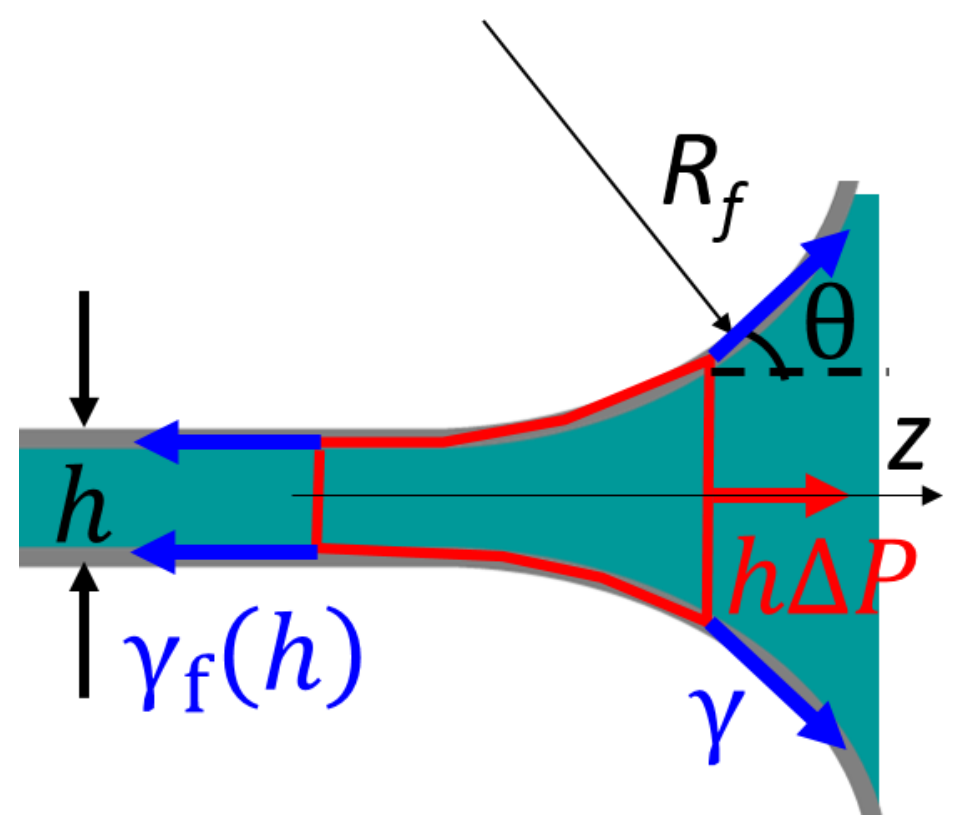

Fig. 4: Schematics of a liquid film between two bubbles of radius $R$ in the foam. The flat part of the film is connected to the Plateau border (meniscus). The larger surface tension in the flat part allows establishment of a mechanical equilibrium although the pressures are not equilibrated. A balance of tensions along the z-axis can be written for instance on the film portion in red.

We now derive the expression of the microscopic length $\alpha$. We consider a liquid film of thickness $h$ of a binary mixture $A / B$, species $A$ having the smallest surface tension, and we derive its thicknessdependent surface tension. We denote $N_{0}$ its total mole number, and $x_{A}^{0}$ and $x_{B}^{0}$ its initial molar fractions. We make the approximation of volume additivity, the total volume of the liquid forming the film $V_{0}$ is thus $V_{0}=\left(x_{A}^{0} v_{A}+x_{B}^{0} v_{B}\right) N_{0}$. When the liquid film forms, $N$ moles occupy its bulk, of respective molar fractions in species $\mathrm{A}$ and $\mathrm{B} x_{A}$ and $x_{B}$, whereas $N_{S}=N_{0}-N$ moles are at the interfaces with air, of respective molar fractions $\Gamma_{A}$ and $\Gamma_{B}$. The bulk volume (without the surface layer) and total surface of the film are respectively

$$
\begin{aligned}
& V=\left(x_{A} v_{A}+x_{B} v_{B}\right) N \\
& S=\left(\Gamma_{A} \sigma_{A}+\Gamma_{B} \sigma_{B}\right) N_{S}
\end{aligned}
$$

Using the conservation equations of species A yields

$$
\Gamma_{A} N_{S}+x_{A} N=x_{A}^{0} \mathrm{~N}_{0}
$$

From eq. (3), (4) and (5) and using $\Gamma_{A}+\Gamma_{A B}=1$, we obtain the following relations

$$
\begin{aligned}
& N_{S}=\frac{S}{\Gamma_{A}\left(\sigma_{A}-\sigma_{B}\right)+\sigma_{B}} \\
& N_{0}=\frac{\mathrm{v}_{0}}{x_{A}^{0}\left(v_{A}-v_{B}\right)+v_{B}} \\
& x_{A}=x_{A}^{0} \frac{\mathrm{N}_{0}}{N}-\Gamma_{A} \frac{N_{S}}{N}
\end{aligned}
$$


From the last equations, we derive the variation in bulk molar fraction resulting from the creation of an interface

$$
x_{A}-x_{A}^{0}=\frac{N_{S}}{\mathrm{~N}_{0}-N_{S}}\left(x_{A}^{0}-\Gamma_{A}\right)
$$

Making the approximation $\mathrm{N}_{0}-N_{S} \approx \mathrm{N}_{0}$ and using the geometrical relation between the total surface of the liquid film and its volume and thickness, $S=2 \mathrm{~V} / \mathrm{h}$, yields

$$
x_{A}-x_{A}^{0}=-\frac{2}{h} \frac{\left(\Gamma_{A}-x_{A}^{0}\right)\left(v_{B}\left(1-x_{A}^{0}\right)+v_{A} x_{A}^{0}\right)}{\left(\Gamma_{A}\left(\sigma_{A}-\sigma_{B}\right)+\sigma_{B}\right)}
$$

Finally, the increase of surface tension of the film of thickness $h$ is obtained from the expansion $\gamma_{F}(h)-\gamma=\left(d \gamma / d x_{A}\right)\left(x_{A}-x_{A}^{0}\right)$ and eq. (10), yielding

$$
\gamma_{f}(h)-\gamma=\frac{2}{h}\left(\frac{d \gamma}{d x_{A}}\right)\left(x_{A}-\Gamma_{A}\right) \frac{\left(v_{B}\left(1-x_{A}\right)+v_{A} x_{A}\right)}{\left(\Gamma_{A}\left(\sigma_{A}-\sigma_{B}\right)+\sigma_{B}\right)}+0\left(\frac{1}{h^{2}}\right)
$$

Where the approximation $x_{A}^{0} \approx x_{A}$ has been made and where values of $\gamma$ and its derivative are taken for $x_{A}=x_{A}^{0}$, i.e. $\gamma=\gamma\left(x_{A}^{0}\right)$ and $\left(d \gamma / d x_{A}\right)=\left(d \gamma / d x_{A}\right)_{x_{A}^{0}}$.

Equation (11) is valid only for thicknesses that are large compared to the molecular sizes, $h \gg v_{i} / \sigma_{i}$. Note that eq. (11) is not based on any thermodynamical hypothesis since it is derived only from molar conservation considerations. For symmetric mixtures, i.e. made of molecules of same molar surface and volume, one obtains from eq. (2) and (11)

$$
\alpha=\frac{2}{\gamma}\left(\frac{\partial \gamma}{\partial x_{A}}\right)\left(x_{A}-\Gamma_{A}\right) \frac{v_{A}}{\sigma_{A}}
$$

Therefore, the ability to foam, as measured by the length $\alpha$, is related to the surface-bulk partition of species $\mathrm{A},\left(x_{A}-\Gamma_{A}\right)$. As a result, with symmetric molecules, foamability is associated with the nonlinearity of surface tension with composition, as previously demonstrated for binary mixtures of different natures ${ }^{7}$.

In asymmetric mixtures, the surface-bulk partition is also at the origin of a thickness-dependent surface tension, as explicit in eq. (11). As in symmetric mixtures, length $\alpha$ and $\left(x_{A}-\Gamma_{A}\right)$ are proportional, but with a - positive - geometric coefficient that depends on $x_{A}$. However, the term $\left(x_{A}-\Gamma_{A}\right)$ is not necessarily associated with a nonlinearity of surface tension. Further discussion on this point requires to examine the variations of surface tension and surface fractions as a function of bulk fractions. We have chosen to use the relation between these quantities known as Butler's equation.

Predictions of Butler's equation. We consider the equation first introduced by Butler ${ }^{2 a}$ that describe the surface/volume partition for ideal mixtures. It has been widely used in the literature, and assumes an ideal behaviour of molecules both in bulk and at interfaces ${ }^{2 b}$. In the picture suggested by Butler, the surface molar fractions follow a Boltzman's law $\Gamma_{i}=x_{i} e^{\frac{\sigma_{i}\left(\gamma-\gamma_{i}\right)}{R T}}$, with $\sigma_{i}\left(\gamma-\gamma_{i}\right)$ the surface energy variation when one mole of species $i$ is displaced from the bulk to the interface, $R$ the ideal gas constant, $T$ the absolute temperature and $\gamma$ the surface tension of the mixture. Since $\Gamma_{A}+\Gamma_{B}=1$, the surface tension $\gamma$ verifies the relation

$$
x_{A} e^{\frac{\sigma_{A}}{R T}\left(\gamma-\gamma_{A}\right)}+\left(1-x_{A}\right) e^{\frac{\sigma_{B}}{R T}\left(\gamma-\gamma_{B}\right)}=1
$$

We introduce several dimensionless parameters: the effective reduced molar surface areas occupied by A and B, respectively $S_{A}=\sigma_{A}\left(\gamma_{B}-\gamma_{A}\right) / R T, S_{B}=\sigma_{B}\left(\gamma_{B}-\gamma_{A}\right) / R T$, and the dimensionless excess surface tension 


$$
E_{\gamma}=\frac{\gamma-\left(\gamma_{A} x_{A}+\gamma_{B} x_{B}\right)}{\gamma_{B}-\gamma_{A}}
$$

This excess value characterizes the deviation from linearity and is positive for superlinear variations of surface tension and negative for sublinear variations. Using these quantities, Butler's equation (eq. (13)) can be written as

$$
x_{A} e^{S_{A}\left(E_{\gamma}+1-x_{A}\right)}+\left(1-x_{A}\right) e^{S_{B}\left(E_{\gamma}-x_{A}\right)}=1
$$

Following eq. (15), the dimensionless excess surface tension $E_{\gamma}\left(x_{A}\right)$ is a function of mixture composition and only depends on two parameters, $S_{A}$ and $S_{B}$. In practice, these parameters are of the order of unity (see Table 1).

We have solved eq. (15) numerically with a typical value of $S_{A}=1$ and different surface ratios $\sigma_{B} / \sigma_{A}=$ $S_{B} / S_{A}$. The corresponding normalized surface tension variations are shown in Fig. $5 \mathrm{a}$ as a function of the bulk molar fraction of the species having the smallest surface tension. For surface ratios ranging from 0.2 to 1 , the variations are sublinear and the nonlinearity increases as the ratio decreases. Conversely, for surface ratios of 3 and 5 , the variations are superlinear and the nonlinearity increases as the ratio increases. However, it does not mean that the species $B$ with the largest surface tension concentrates at the surface. From the computed values of $\gamma\left(x_{A}\right)$, we also determine $\Gamma_{A}\left(x_{A}\right)$ in Fig. $5 \mathrm{~b}$ : Clearly, whatever the surface ratio of the molecules, the molar fraction in species $A$ at the surface is larger than the one in the bulk. The associated nonlinearity increases monotonically with the surface ratio of the molecules.

This result can be understood by expanding eq. (15) for $S_{A}$ and $S_{B} \ll 1$, in the limit of an entropydominated partition between surface and bulk. In that case, the dimensionless excess surface tension is given by

$$
E_{\gamma}=\frac{x_{A}\left(1-x_{A}\right)}{x_{A}+\frac{S_{B}}{S_{A}}\left(1-x_{A}\right)}\left(\frac{s_{B}}{s_{A}}-1\right)
$$

The ratio in the right-hand term of eq. (16) is positive. Therefore, the sign of the nonlinearity is given by the sign of $\left(\frac{S_{B}}{S_{A}}-1\right)$. This result is in qualitative agreement with the data of Fig. 5 for surface ratios different from unity, for which a sublinear behaviour is reported for ratios of 0.2 and 0.5 (i.e. $S_{B} / S_{A}<$ 1 ) and a superlinear behaviour for ratios of 3 and $5\left(S_{B} / S_{A}>1\right)$. In contrast, while the approximated eq. (16) predicts the surface tension to vary linearly for a ratio of 1 (i.e. $E_{\gamma}=0$ ), full resolution of Butler's equation with the parameters of Fig. 5 yields sublinear variations for the latter ratio. Instead a linear behaviour is predicted for a surface ratio close to 2 according to the complete Butler's equation. The contribution of surface energies, which are assumed to be very small in the derivation of eq. (16), must be considered to understand the latter results. 

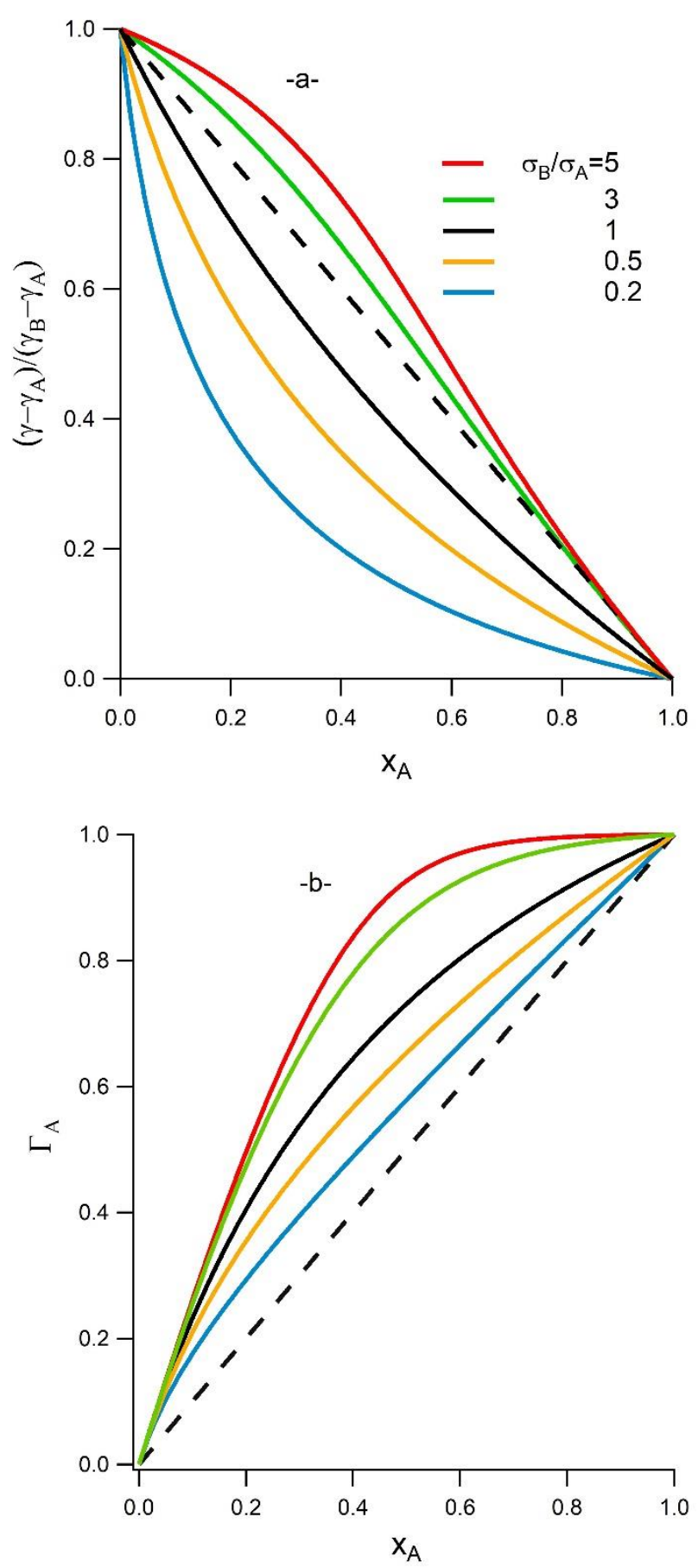

Fig. 5: (a) Normalised surface tensions as a function of the bulk molar fraction of species $A$, predicted by Butler's equation (eq. (14)) with arbitrarily chosen values: $S_{A}=1$ and different $S_{B} / S_{A}$ ratios. (b) Corresponding surface molar fraction of species $A$.

Actually, the surface tension of the mixture is determined by the product of molecular surfaces and the difference between the surface energies. If the surface energies of the two molecules are close, their partitions between surface and bulk are also similar. As a result, if the species with the largest surface tension has also the largest molar surface, its contribution to surface tension will be proportional to its concentration in bulk times its molar surface, leading to superlinear variations of surface tension. This limit is predicted by eq. (16). However, if the difference between surface energies is large, this effect is counterbalanced by the fact that a smaller surface concentration of the species with the larger surface energy is favoured, as compared to bulk. These two effects (ratio between 
occupied surfaces and partition) are of the same order of magnitude because the value of the effective reduced molar surface is of the order of unity. This explains why the surface tension of a mixture varies superlinearly only if the species with the largest surface tension also has the largest molar surface, and if the ratio of the molar surfaces of the two species exceeds some critical value. The crossover between sub and superlinear variations of the interfacial tension can be found by solving Butler's equation for $E_{\gamma}=0$ and $x_{A}=1 / 2$. The following relation is obtained

$$
S_{B}=-2 \ln \left(2-e^{S_{A} / 2}\right)
$$

which yields $S_{B} / S_{A}=2.09$ for $S_{A}=1$. Therefore, the crossover between super and sub linear superficial tension depends in a subtle way on the respective values of the molar surfaces and on the difference between surface energies. Sublinearity is favored by large differences in surface energies whereas difference of molar surfaces are at the origin of either superlinear or sublinear behaviors, depending on the surface ratio. Similar conclusions were drawn by Prigogine and his co-workers for non-ideal solutions ${ }^{12}$. They in particular showed that, in addition to surface ratio, the heat of mixing contributes to lower surface tension of mixtures.

We now compare the predictions of Butler's equation with the experimental data of Fig. 2. For each mixture, we have reported the computed surface tensions in Fig. 2. The molar surfaces are unknown; a usual approximation made in the literature considers cuboid molecules, allowing computation of the molar surfaces from the molar volumes. However, we have found the experimental surface tensions are not described using values for molar surfaces obtained in this approximation; a correct description requires more asymmetric molar surfaces as can be seen in Table 1, in which we report the value of surface ratios in best agreement with the experimental data. In each case, the molar surface of species A was arbitrarily chosen to be the one given by the cuboid approximation, and the surface ratio was adjusted in order to match the surface tension curves following eq. (13).

Numerous attempts have been made to provide a quantitative description of the surface tension of mixtures as a function of their composition. ${ }^{2 c}, 2 \mathrm{e}, 3 \mathrm{a}, 11 \mathrm{a}, 11 \mathrm{~d}, 12,15$ However, because of the missing information on the molecular surface, there is no available model valid whatever the nature of the mixture. As reported, molar surfaces of a given molecule may vary from one mixture to another, depending on the nature of the molecule it is mixed with. For a given mixture, the molar surfaces may as well vary with composition. In addition, nonideal behavior in bulk but also at the surfaces can induce deviations from Butler's equation. We have not tried to provide a more quantitative description of the surface tensions of the mixtures investigated since we focus here on the link between surface tension nonlinearity and foamability.

Microscopic length $\alpha$ of the studied mixtures We establish the expression for $\alpha$ in the framework of ideal mixtures. The derivative of surface tension $\left(\frac{\partial \gamma}{\partial x_{A}}\right)$ is readily obtained by computing the derivative of Butler's equation (eq. (13)) with respect to $x_{A}$

$$
\frac{\partial \gamma}{\partial x_{A}}=\frac{R T\left(x_{A}-\Gamma_{A}\right)}{\left(1-x_{A}\right) x_{A}\left(\Gamma_{A} S_{A}+\left(1-\Gamma_{A}\right) S_{B}\right)}
$$

Equation (11) giving the thickness-dependent surface tension then becomes

$$
\gamma_{f}(h)-\gamma=\frac{1}{h} \frac{R T\left(\Gamma_{A}-x_{A}\right)^{2}}{2\left(1-x_{A}\right) x_{A}} \frac{\left(v_{B}\left(1-x_{A}\right)+v_{A} x_{A}\right)}{\left(\Gamma_{A} \sigma_{A}+\left(1-\Gamma_{A}\right) \sigma_{B}\right)^{2}}+0\left(\frac{1}{h^{2}}\right)
$$

Finally, the expression for the microscopic length $\alpha$ - in the case of ideal solutions - characterizing the ability to form foams is deduced from eq. (18) and (2) 


$$
\alpha=\frac{R T}{\gamma} \frac{\left(\Gamma_{A}-x_{A}\right)^{2}}{\left(1-x_{A}\right) x_{A}} \frac{\left(v_{B}\left(1-x_{A}\right)+v_{A} x_{A}\right)}{2\left(\Gamma_{A} \sigma_{A}+\left(1-\Gamma_{A}\right) \sigma_{B}\right)^{2}}
$$

Mixtures with identical bulk and surface compositions, for which $\Gamma_{A}=x_{A}$ are characterized by $\alpha=0$, meaning that foams are as unstable as in pure liquids for which bubble lifetimes are of the order of $1 \mathrm{~ms}$. However, as emphasized above, the species with the smallest surface tension is always more concentrated at the surface than in the bulk, i.e. $\Gamma_{A}-x_{A}>0$ whatever the surface ratio of molecules. As a result, provided the surface tensions are different, the microscopic length is non zero and foam lifetimes are enhanced.

We have computed the variations of length $\alpha$ following eq. (19) and they are reported as a function of mixture composition in Fig. 6. Cuboid molecules have been considered in order to determine the molar surfaces, with surface ratios close to the ones determined for the used liquid mixtures (see Table 1). The length $\alpha$ is at most of a molecular size. Similarly to the variation of $L_{\tau}$ shown in Fig. 3, all curves for length $\alpha$ exhibit a maximum at a composition that depends on the asymmetry of the mixture; in addition, the position of the maximum is shifted toward the larger molar fractions in species $A$ as the surface ratio increases, as found for $L_{\tau}$. A qualitative correlation between the two lengths is therefore observed.

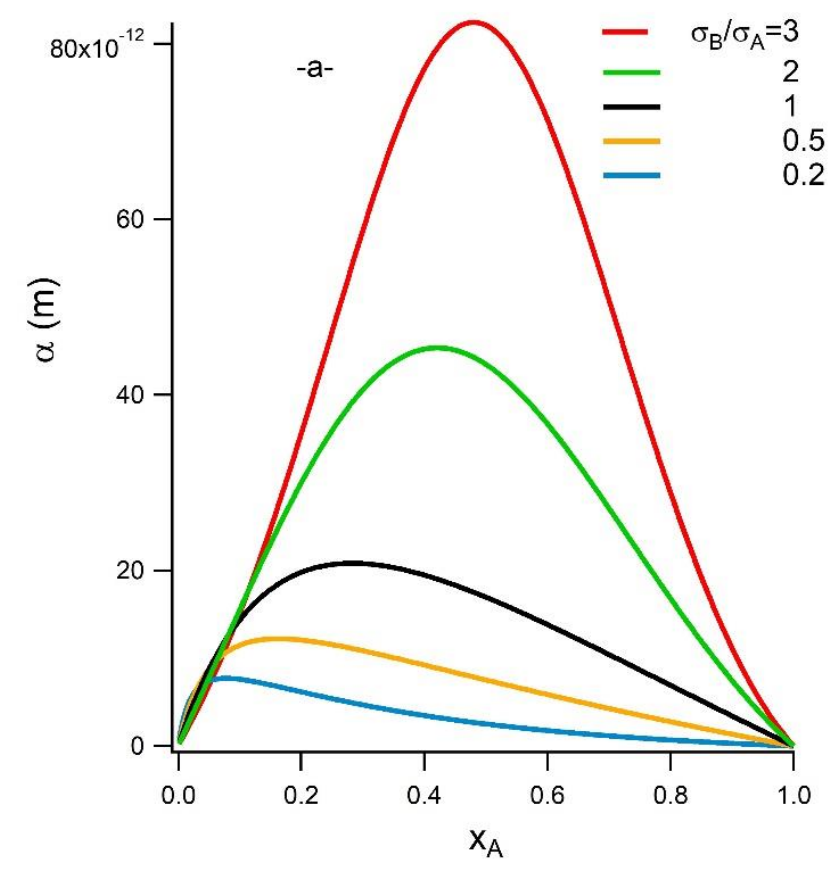




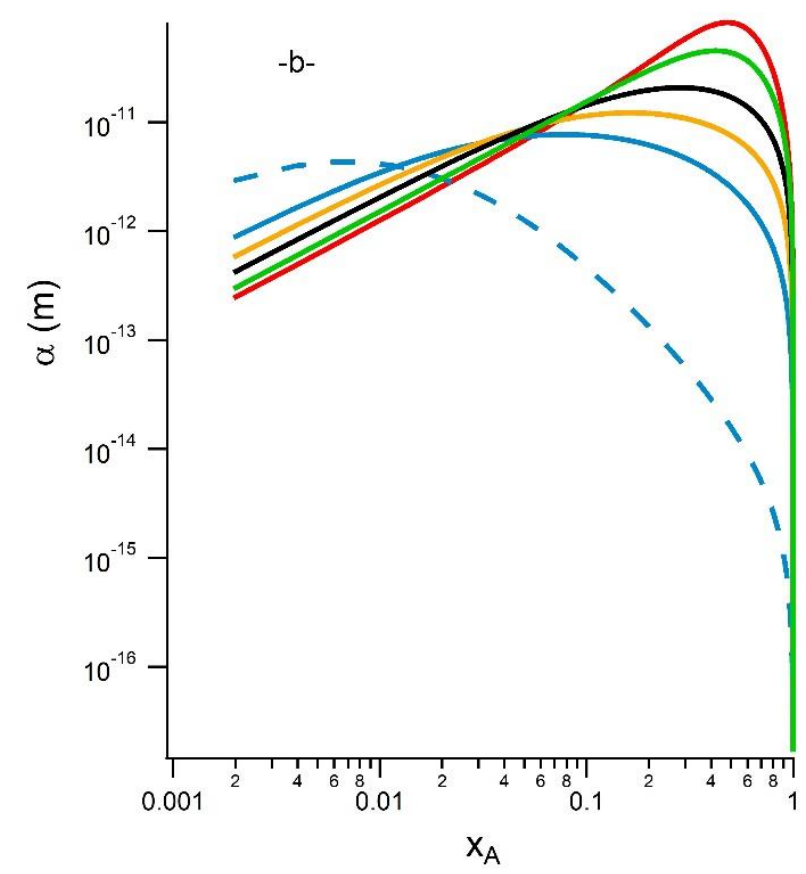

Fig. 6: Length $\alpha$ characterizing the increase of surface tension with decreasing thickness of a film of binary mixture, as a function of the mixture composition in linear (a) and log (b) plots. The curves were computed using eq. (19), derived in the case of ideal solutions. The additional dashed line in (b) was obtained with an volume ratio of $10^{-2}$.

However, the amplitudes of length $\alpha$ and $L_{\tau}$ vary differently with the volume ratio. In particular, we have found the PDMS/decane mixture formed foams with very large lifetimes yielding very large values of $L_{\tau}$. The position of the maximum in the experimental curve is also reached for a strikingly small $\left(\sim 10^{-3}\right)$ molar fraction in PDMS. As reported in Fig. 6 (dashed line), such a value corresponds to a surface ratio close to $10^{-2}$, much smaller than the one determined from surface tension variations. Cleary, eq. (19) derived in the framework of ideal mixtures does not capture the effects at stake in this mixture. A more quantitative description of the foaming properties would require to consider non ideal behaviors, i.e. fugacities of the different species in bulk and at the interfaces. Nevertheless, experimental determination of the fugacity of molecules at an interface is difficult and molecular dynamics simulations are more likely to help bringing new light on the link between species partition and foamability. ${ }^{16}$

\section{CONCLUSION}

We have shown previously that the foams formed in liquid mixtures of molecules with similar surfaces have lifetimes three to four order of magnitude larger than the ones formed in pure liquids, and that it was a consequence of the sublinear variations of their surface tension with composition. Here we demonstrate that the case of mixtures of molecules with different sizes is more complex. We show that in the case of ideal solutions, the surface tension may vary superlinearly with composition, provided the species with the largest surface energy has a large enough molar surface, consistently with our experimental findings on mixtures with different surface ratios. However, whatever the sign of the nonlinearity, the surface concentration of the species with the smallest surface energy is always larger than the bulk one; we show the ability to foam originates from this concentration difference, and confirm that finding experimentally. As a result, mixtures of species of asymmetric molecular sizes 
may exhibit either sub or superlinear variations of their surface tension with composition and nonetheless have a stronger ability to foam than pure liquids.

\section{References}

1. von Szyszkowski, B., Experimental studies of the capillary properties of aqueous solutions of fatty acids. Zeitschrift für Physikalische Chemie 1908, 64, 385-414.

2. (a) Butler, J. A. V., The thermodynamics of the surfaces of solution. Proceedings of the Royal Society of London Series a-Containing Papers of a Mathematical and Physical Character 1932, 135 (827), 348-375; (b) Eriksson, J. C., On the thermodynamics of surface systems. Advances in Chemical Physics 1964, 6, 145-174; (c) Eberhart, J. G., The Surface Tension of Binary Liquid Mixtures. J. Phys. Chem 1966, 70 (4), 1183-1186; (d) Guggenheim, E. A.; Adam, N. K., The thermodynamics of adsorption at the surface of solutions. Proceedings of the Royal Society of London Series a-Containing Papers of a Mathematical and Physical Character 1933, 139 (837), 218-236; (e) Belton, J. W.; Evans, M. G., STUDIES IN THE MOLECULAR FORCES INVOLVED IN SURFACE FORMATION .2. THE SURFACE FREE ENERGIES OF SIMPLE LIQUID MIXTURES. Transactions of the Faraday Society 1945, 41 (1), 1-12. 3. (a) Santos, M. S. C. S.; Reis, J. C. R., Partial molar surface areas in liquid mixtures. Theory and evaluation in aqueous ethanol. Journal of Molecular Liquids 2019, 273, 525-535; (b) Shardt, N.; Wang, Y.; Jin, Z.; Elliott, J. A. W., Surface tension as a function of temperature and composition for a broad range of mixtures. Chemical Engineering Science 2021, 230, 116095.

4. (a) Chen, H.; Gan, W.; Lu, R.; Guo, Y.; Wang, H. F., Determination of structure and energetics for Gibbs surface adsorption layers of binary liquid mixture 2. Methanol plus water. J. Phys. Chem. $B$ 2005, 109 (16), 8064-8075; (b) Kirschner, J.; Gomes, A. H. A.; Marinho, R. R. T.; Bjorneholm, O.; Agren, H.; Carravetta, V.; Ottosson, N.; de Brito, A. N.; Bakker, H. J., The molecular structure of the surface of water-ethanol mixtures. Physical Chemistry Chemical Physics 2021, 23 (19), 11568-11578. 5. Lhuissier, H.; Villermaux, E., Bursting bubble aerosols. Journal of Fluid Mechanics 2012, 696, 5-44.

6. (a) Ross, S.; Nishioka, G., Foaminess of binary and ternary solutions. Journal of Physical Chemistry 1975, 79 (15), 1561-1565; (b) Tuinier, R.; Bisperink, C. G. J.; vandenBerg, C.; Prins, A., Transient foaming behavior of aqueous alcohol solutions as related to their dilational surface properties. Journal of Colloid and Interface Science 1996, 179 (2), 327-334.

7. Tran, H. P.; Arangalage, M.; Jorgensen, L.; Passade-Boupat, N.; Lequeux, F.; Talini, L., Understanding Frothing of Liquid Mixtures: A Surfactantlike Effect at the Origin of Enhanced Liquid Film Lifetimes. Physical Review Letters 2020, 125 (17), 178002.

8. Gaines, G. L., SURFACE TENSION OF POLYMER SOLUTIONS .I. SOLUTIONS OF POLY(DIMETHYLSILOXANES). Journal of Physical Chemistry 1969, 73 (9), 3143-\&.

9. Kendall, J.; Monroe, K. P., The viscosity of liquids. Il. The viscosity-composition curve for ideal liquid mixtures. Journal of the American Chemical Society 1917, 39, 1787-1802.

10. Bikerman, J. J., Measurement of Foaminess In Foams. Springer-Verlag: New York, 1973.

11. (a) Suarez, J. T.; Torresmarchal, C.; Rasmussen, P., PREDICTION OF SURFACE TENSIONS OF NONELECTROLYTE SOLUTIONS. Chemical Engineering Science 1989, 44 (3), 782-786; (b) Nath, S., Surface tension of nonideal binary liquid mixtures as a function of composition. Journal of Colloid and Interface Science 1999, 209 (1), 116-122; (c) Kahl, H.; Wadewitz, T.; Winkelmann, J., Surface tension of pure liquids and binary liquid mixtures. Journal of Chemical and Engineering Data 2003, 48 (3), 580-586; (d) Kaptay, G., Partial Surface Tension of Components of a Solution. Langmuir 2015, 31 (21), 5796-5804; (e) Santos, M. S. C. S.; Reis, J. C. R., Thermodynamic evaluation of molar surface area and thickness of water plus ethanol mixtures. Journal of Molecular Liquids 2018, 255, 419-428.

12. Prigogine, I.; Marechal, J., THE INFLUENCE OF DIFFERENCES IN MOLECULAR SIZE ON THE SURFACE TENSION OF SOLUTIONS .4. Journal of Colloid Science 1952, 7 (2), 122-127.

13. Pugh, R. J., Foaming, foam films, antifoaming and defoaming. Advances in Colloid and Interface Science 1996, 64, 67-142. 
14. Koehler, S. A.; Hilgenfeldt, S.; Weeks, E. R.; Stone, H. A., Drainage of single Plateau borders: Direct observation of rigid and mobile interfaces. Physical Review E 2002, 66 (4), 04601 (R).

15. Santos, M. S. C. S.; Reis, J. C. R., New Thermodynamics for Evaluating the Surface-phase Enrichment in the Lower Surface Tension Component. Chemphyschem 2014, 15 (13), 2834-2843.

16. (a) Matsumoto, M.; Takaoka, Y.; Kataoka, Y., Liquid vapor interface of water-methanol mixture .1. Computer-simulation. Journal of Chemical Physics 1993, 98 (2), 1464-1472; (b) Chang, T. M.; Dang, L. X., Liquid-vapor interface of methanol-water mixtures: A molecular dynamics study. J. Phys. Chem. B 2005, 109 (12), 5759-5765.
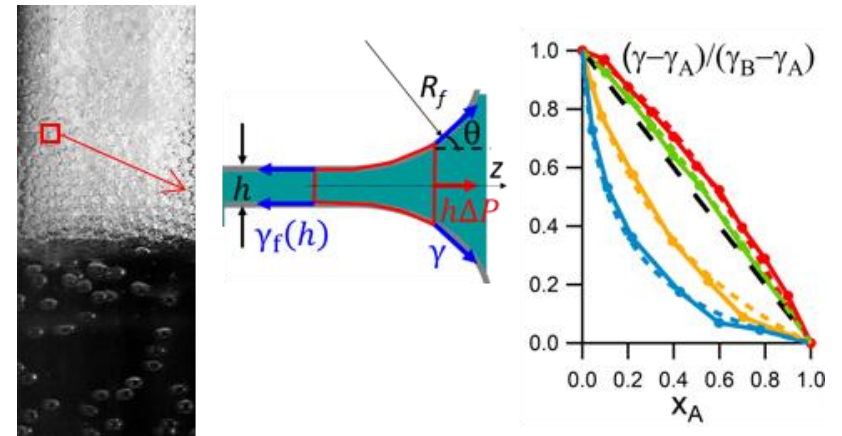

For Table of Contents Only 